\title{
Component Change of Urine Crystallites with Placement Time in Patients of Calcium Oxalate Stone Patients and Control Subjects
}

\author{
Xiao-Ling Wen \\ Institute of Biomineralization and Lithiasis Research \\ Jinan University \\ Guangzhou 510632 \\ Email: zwenxiaoling@163.com
}

Xin-Yuan Sun

Institute of Biomineralization and Lithiasis Research

Jinan University

Guangzhou 510632

Email: sunxinyuan1985@163.com

\author{
Jian-Ming Ouyang* \\ Institute of Biomineralization and Lithiasis Research \\ Jinan University \\ Guangzhou 510632 \\ Email: toyjm@jnu.edu.cn
}

\begin{abstract}
Purpose] To study the component change of urine crystallites with different sizes in the urines of calcium oxalate $(\mathrm{CaOx})$ calculi patients and healthy controls with placement time. [Methods] A combination of high-resolution transmission electron microscopy (HRTEM), fast Fourier transformation (FFT), energy dispersive X-ray spectroscopy (EDS) and X-ray diffraction (XRD) was performed to detect the component of urinary crystallites. Different sizes of urine crystallites were obtained after filtration of urine through microporous membrane with different pore sizes $(0.22,0.45$, $1.2,3$, and $8 \mu \mathrm{m})$. [Results] The main components of urine crystallites in patients with $\mathrm{CaOx}$ calculi are calcium oxalate monohydrate (COM), uric acid, and calcium phosphate. As the placement time increases, the deposition quality of crystallites increased. TEM analysis showed that the amount of small-sized urine crystallites (approximately $100 \mathrm{~nm}$ ) in the healthy subjects was significantly higher than that of the patients. However, the number of large micron-scale crystals in the controls was reduced remarkably. [Conclusion] The rapid aggregation of urine crystallites may be an important factor affecting the growth of crystallites in $\mathrm{CaOx}$ calculi patients. The increase of $\mathrm{COM}$ in patient's urine was crucial factors affecting the formation of uroliths.
\end{abstract}

Keywords: Urine nanocrystallite; urine component; HRTEM; EDS

\section{INTRODUCTION}

The occurrence of urolithiasis is still not effectively prevented and its mechanism is still unclear $[1,2]$. Several studies have investigated the presence of crystallites in the urine [2-7]. Most of them believe that urine crystallites are highly indicative of stone disease activity in lithogenic patients and predictive of stone recurrence. Crystallites with a size larger than $12 \mu \mathrm{m}$ in lithogenic urines reportedly accounted for $16 \%$ to $65 \%$ of the urinary crystals, while those in healthy urine accounted for less than $13 \%$. Small particles with a size less than $20 \mu \mathrm{m}$ can easily be excreted in the urine, whereas larger crystallites tend to precipitate in the renal tubule or in the narrow position of the urethra and eventually form urinary stones. Werness et al. [8] studied the urinary crystals of 162 cases of healthy controls and 4835 cases of urolithiasis patients and found that the number of urinary crystals in patients was greater than that of the controls, but reached a normal level after drug treatment.

The formation of stones is reportedly related to the process of nucleation, growth, aggregation, and cell adhesion of urine crystallites [9]. Urines are believed to contain various sizes of crystallites. Over the past ten years, we hope to develop a reliable method to diagnose kidney stones by urine examination. If a certain property of urine crystallites was a reliable predictor of disease, such a method would represent a non-invasive method that could potentially by applied.

This study obtained different sizes of urine crystallites after filtration of urine through microporous membrane with different pore sizes $(0.22,0.45,1.2,3$, and $8 \mu \mathrm{m})$ and studied the composition change in the different sizes of urine crystallites in order to further illustrate the relation between the size of urine crystallites and the formation of urolithiasis.

\section{EXPERIMENTAL SECTION}

\section{A. Reagents and instruments}

Absolute ethanol, sodium azide $\left(\mathrm{NaN}_{3}\right)$ and all the reagents were analytical purity. Double distilled water was used. Microporous membrane (pore size: 0.22, 0.45, $1.2,3$ and $8 \mu \mathrm{m}$, respectively) was purchased from Xinya company (Shanghai, China).

HRTEM was conducted on a HRTEM (JOEL 2100F) with a maximum acceleration voltage of $200 \mathrm{kV}$. To determine the morphology, component, element, and crystal structure of urine nanocrystallites, we performed SEAD and EDS of the HRTEM, and fast Fourier transformation (FFT) analysis in the Digital Micrograph software was also conducted to obtain the patterns. 
$\mathrm{X}$-ray diffraction $(\mathrm{XRD})$ results were recorded on a $\mathrm{D} / \max -\gamma \mathrm{A}$ X-ray diffractometer (Rigaku, Japan). Centrifugalization was carried by $80-1$ sedimentation centrifuge (Shanghai Surgical Instrument Factory, China). Ultrasonication was carried out using KQ3200 DE type ultrasonic instrument (Kunshan Ultrasonic Instrument Company, China).

\section{B. Collection and treatment of urine}

30 samples of fresh morning urine were collected, among them 10 samples were randomly healthy subjects with no history of urolithiasis ( 7 men and 3 women; mean age $=37.2$ years; range $=22 \sim 71$ years), and 20 samples were the patients with $\mathrm{CaOx}$ stones ( 12 men and 8 women; mean age $=42.7$ years; range $=25 \sim 67$ years). The calculi patients were from the Lithotripsy Center of the First Affiliated Hospital of Jinan University, 10 healthy subjects were from the students and teachers of Jinan University, all of them are Chinese. The study was approved by the Institutional Review Board of the First Affiliated Hospital of Jinan University, and all participants provided informed consent.

\section{XRD detection of urinary nanocrystallites}

Fasting morning urines were collected. The $\mathrm{pH}$ value was detected, and $2 \% \mathrm{NaN}_{3}$ solution $(10 \mathrm{~mL} / \mathrm{L}$ urine sample) was added to these urine samples as antiseptic. Anhydrous alcohol was added to the urine sample [V (urine): $\mathrm{V}$ (ethanol) = 3:2] to denature the proteins. After the urine sample was stirred for 3 min and left undisturbed for half an hour, the urine proteins and the cell debris were removed by centrifugation at $4000 \mathrm{r} / \mathrm{m}$ for $15 \mathrm{~min}$. The liquid supernatant became clear and then was filtered by microporous membrane with different pore sizes $(0.22$, $0.45,1.2,3$, and $8 \mu \mathrm{m}$, respectively). The filtered urine was stored in clean glassware for examination.

\section{Effects of placement time on components of crystallites}

After $3 \mathrm{~min}$ of ultrasonication of the urine sample filtered through the microporous membrane with different pore sizes, XRD detection was performed at placement time $\mathrm{t}=0,1,2,4$, and $8 \mathrm{~h}$.

\section{E. HRTEM, FFT and EDS analyses of urinary naocrystallites}

Approximately $5 \mu \mathrm{L}$ of urine was submerged in a copper mesh by a microsyringe, the urine was preliminarily dried using an absorbent paper from the back of the mesh so as to remove most of the water in urine. After such a treatment, most of the soluble salts (such as $\mathrm{NaCl}$ and urea) in urine were sucked off with the urine by the paper. Then the mesh was stored in a desiccator for $2 \mathrm{~d}$ prior to HRTEM, FFT, and EDS analyses.

\section{RESULTS AND DISCUSSION}

\section{A. TEM observation of various sizes of urine crystallites with placement time $(t)$}

Figs. 1 and 2 showed the TEM images of the urine crystallites of the representative $\mathrm{CaOx}$ calculi patients and healthy controls with placement time $(t)$ after filtration through a 0.22 and $1.2 \mu \mathrm{m}$ microporous membrane. The results were as follows: (1) The aggregation degree increased with increased $t$. (2) The structures of the urine crystallite aggregates of the controls were looser (Fig. 3), whereas those of the patient's crystallite aggregates were much denser (Figs. 1 and 2). In particular, the urine crystallites of the lithogenic patients were easier to aggregate than those of the controls.

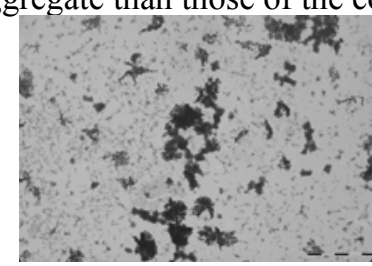

(a)
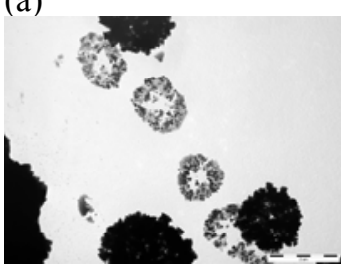

(c)

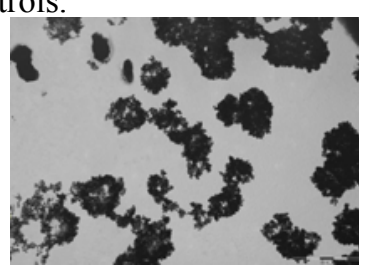

(b)
Figure 1. TEM images of urine crystallites of the representative $\mathrm{CaOx}$ calculi patients with placement time after filtration through a $0.22 \mu \mathrm{m}$ microporous membrane. (a) 0; (b) 1; (c) 4 h. Bars: $500 \mathrm{~nm}$

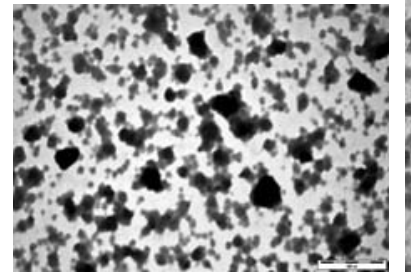

(a)

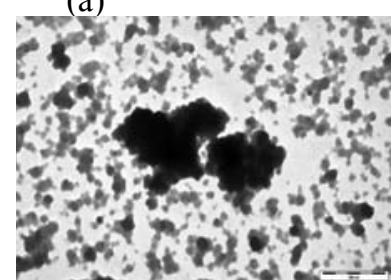

(c)

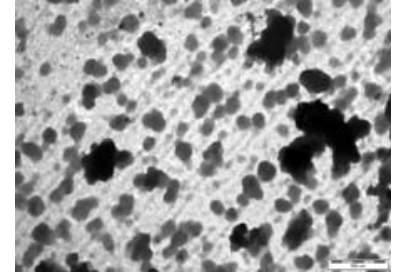

(b)
Figure 2. TEM images of urine crystallites of the representative $\mathrm{CaOx}$ calculi patients with placement time after filtration through a $1.2 \mu \mathrm{m}$ microporous membrane. (a) 0; (b) 1; (c) 4 h. Bars: $500 \mathrm{~nm}$

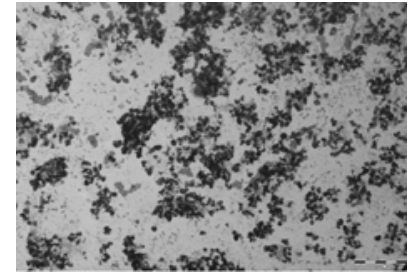

(a)

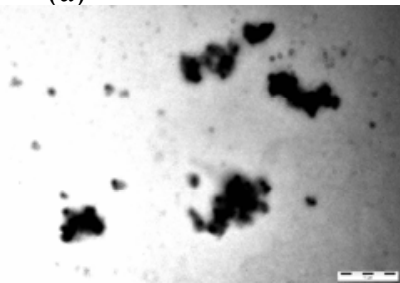

(c)

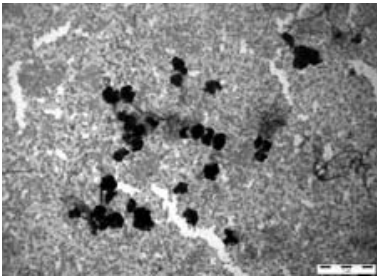

(b)
Figure 3. TEM images of urine crystallites of the representative healthy controls with placement time after filtration through a $1.2 \mu \mathrm{m}$ microporous membrane. (a) 0; (b) 1; (c) $4 \mathrm{~h}$. Bars: $1 \mu \mathrm{m}$ 


\section{B. Component change of urine crystallites with placement time}

XRD was used to study the component change of urine crystallites of 20 stone patients with placement time $(t)$. Figs. 4 and 5 showed some examples. It can be seen that:

1) As the placement time increases, the intensity of diffraction peaks increased, but the composition of crystallites did not change obviously. It indicated that the deposition of quality crystallites increased with increased placement time. As shown in Fig. 4, the main components of urine crystallites were UA and COM crystals, and the composition of crystallites did not change obviously with increasing placement time after placed for $2 \mathrm{~h}$ and $8 \mathrm{~h}$. However, the diffraction peak intensity of the crystallites increased as the placement time increases. After placement for $8 \mathrm{~h}$, the intensity of diffraction peaks at $\mathrm{d}=$ $3.93 \AA((\overline{2} 11)$ plane of UA) and 2.98, $2.49 \AA((\overline{2} 02)$ and (112) planes of COM) increased [10] (Figs. $4 \mathrm{a} \& 4 \mathrm{~b}$ ). The increased intensity contributed to the increased deposition of quality crystallites with increased placement time.

As shown in Fig. 4c and 4d, the increased amplitude of COM peak was larger than that of UA, namely, the $\mathrm{COM} / \mathrm{UA}$ ratio increased with increasing placement time.

2) With the increase of placement time (t), COD gradually disappeared, and COM increased gradually, it was attributed to the thermodynamically unstable COD easily converting into COM.

For example, after filtration through a $0.45,1.2$ and 3 $\mu \mathrm{m}$ microporous membrane, respectively, we detected the diffraction peak at $\mathrm{d}=8.63 \AA$ which assigned to $(110)$ plane of COD; diffraction peak at $\mathrm{d}=3.93 \AA$ which assigned to uric acid; diffraction peaks at $\mathrm{d}=2.79,1.98 \AA$ which assigned to COM. After placement for $8 \mathrm{~h}$, the diffraction peak of COD disappeared, while the diffraction peak of COM appeared (Fig. 5).
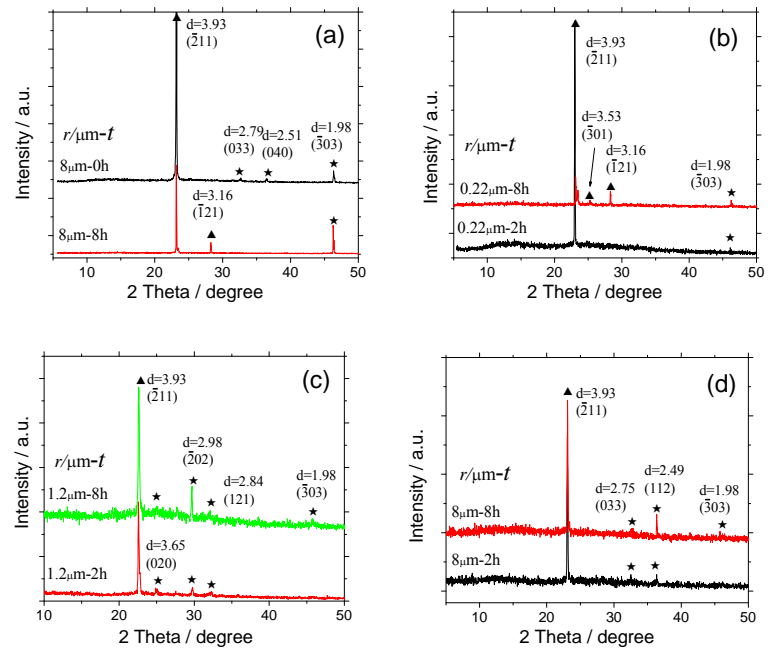

Figure 4. XRD patterns of urine crystallites of $\mathrm{CaOx}$ calculi patients with increasing placement time $(\mathrm{t})$ after filtration through microporous membrane with different pore size (r). $\star$ : COM; $\mathbf{\Delta}$ : uric acid; $九$ : COD.
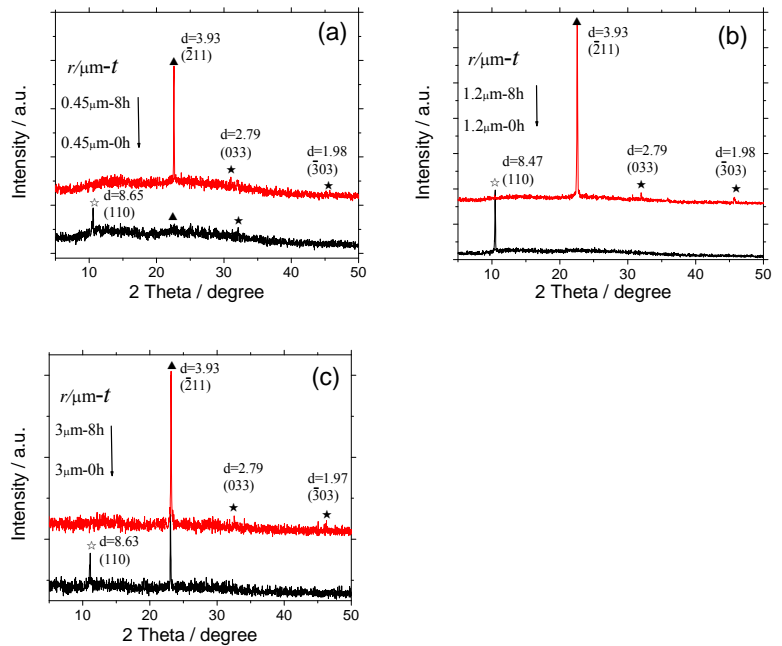

Figure 5. XRD patterns of urine crystallites of $\mathrm{CaOx}$ calculi patients with increasing placement time after filtration through a microporous membrane of $0.45,1.2$, and $3 \mu \mathrm{m}$, respectively. $\mathrm{t}$ : placement time; $\mathrm{r}$ : microporous membrane pore size. $\star$ : COM; $\boldsymbol{\Delta}$ : uric acid; $\_$: COD.

\section{Component study by HRTEM, FFT, and EDS}

Calcium oxalate stones often have a core-shell structure. Previous reports usually detected the components and elements on the surface and interior layer of stones by FT-IR, SEM-EDAX and TGA etc., and then predicted the formation mechanism of stones based on the difference of these two components. For example, Fazil Marickar et al [11] reported that since the main component in interior layer of stone was $\mathrm{CaOx}$ and $\mathrm{CaP}$, whereas that on the surface was calcium oxalate monohydrate (COM), thus deduced that $\mathrm{CaP}$ crystals induced the development of COM crystals and finally the formation of COM stones by heterogeneous nucleation. In vitro simulation experiment, $\mathrm{CaP}$ and uric acid (UA) crystals induced the development of COM crystals by heterogeneous nucleation was also reported in literature.

High-resolution transmission electron microscopy (HRTEM), fast Fourier fransformation (FFT) and energy dispersive X-ray spectroscopy (EDS) were used to further characterize the components of urine crystallites. Fig. 6 showed the images of HRTEM and FFT in different areas of urinary crystallites of patients with $\mathrm{CaOx}$ calculi. To analyze the clear lattice fringes by random selection, we detected the lattice fringe at $d=3.65 \AA$ in Fig. $6 a$ and at $d$ $=3.24 \AA$ in Fig. $6 \mathrm{~b}$, which were assigned to (020) plane of $\mathrm{COM}(d=3.65 \AA)$ and $(021)$ plane of UA $(d=3.23 \AA)$, respectively [10]. That is, the main components of urinary crystallites of patients with $\mathrm{CaOx}$ calculi were $\mathrm{COM}$ and UA, which was consistent with XRD result.

To further study the components of urinary crystallites, EDS of the urinary crystallites of patients with $\mathrm{CaOx}$ calculi was performed. Fig. 7 shows the representative EDS distribution. The absorption peaks of $\mathrm{C}, \mathrm{O}, \mathrm{Ca}, \mathrm{P}$ element were detected, indicating the presence of $\mathrm{CaOx}$ and calcium phosphate $(\mathrm{CaP})$. $\mathrm{N}$ was not detected. Thurgood et al. [12] also detected the characteristic absorption peaks of $\mathrm{C}$ and $\mathrm{O}$ of UA crystals in urine by EDS analysis, but $\mathrm{N}$ was not detected. 

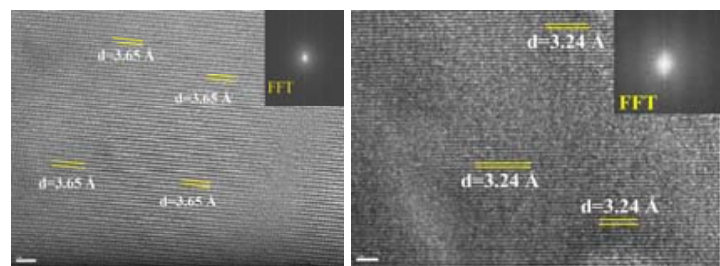

Figure 6. FFT images of HRTEM in different areas of urinary naocrystallites of $\mathrm{CaOx}$ stone patients.
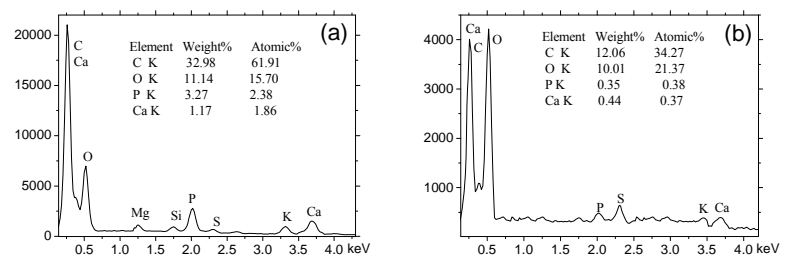

Figure 7. EDS analysis of the elemental distribution of urinary crystallites of $\mathrm{CaOx}$ stone patients.

\section{CONCLUSIONS}

XRD, FFT, and EDS results indicated that the main components of urine nanocrystallites in patients with $\mathrm{CaOx}$ calculi were UA, COM, and $\mathrm{CaP}$. That is, the formation of $\mathrm{CaOx}$ calculi was closely related to the presence of $\mathrm{UA}$ and $\mathrm{CaP}$ crystallites in urine. As the placement time $(t)$ increased, the intensity of diffraction peaks increased, indicating that the deposition of quality crystallites increased with increased placement time. Sometimes, COD gradually disappeared, and COM increased gradually with $t$ increased, it was attributed to the thermodynamically unstable COD easily converting into COM. Urine crystallites are highly indicative of stone disease activity in lithogenic patients and predictive of stone recurrence

\section{ACKNOWLEDGMENTS}

This research work was supported by the Natural Science Foundation of Guangdong Province of China and the Natural Science Foundation of China (81170649).

\section{CONFLICTS OF INTEREST}

The authors declare that there is no conflict of interests regarding the publication of this article.

\section{REFERENCES}

[1] B. Grohe, S. Hug, A. Langdon, J. Jalkanen, K. A. Rogers, H. A. Goldberg, M. Karttunen, G. K. Hunter. Mimicking the biomolecular control of calcium oxalate monohydrate crystal growth: The effect of contiguous glutamic acids. Langmuir, 28(2012), 12182-12190.

[2] H. Brzica, D. Breljak, B. C. Burckhardt, G. Burckhardt, I. Sabolic. "Oxalate: From the Environment to Kidney Stones," Arch. Ind. Hygiene Toxicol., 64(2013), 609-630.

[3] R. Dyer, B. E. Nordin. Urinary crystals and relation to stone formation. Nature, 215(1964), 751-752.

[4] W. G. Robertson, M. Peacock, B. E. C. Nordin. Calcium crystalluria in recurrent renal-stone formers. Lancet, 2(1969), 21 24.

[5] M. Daudon, C. Hennequin, G. Boujelben, B. Lacour, P. Jungers. Serial crystalluria determination and the risk of recurrence in calcium stone formers. Kidney Int., 67(2005), 1934-1943.

[6] G. B. Fogazzi. "Crystalluria: a neglected aspect of urinary sediment analysis,” Nephrol. Dial. Transplant, 11(1996), 379-387.

[7] M. Daudon, P. Jungers. "Clinical value of crystalluria and quantitative morphoconstitutional analysis of urinary calculi," Nephron Physiol., 98(2004), 31-36.

[8] P. G. Werness, J. H. Bergert, L. H. Smith. "Crystalluria,” J. Cryst. Growth, 53(1981), 166-181.

[9] D. S. Li, M. H. Nielsen, J. R. I. Lee, C. Frandsen, J. F. Banfield, J. J. De Yoreo. "Direction-specific interactions control crystal growth by oriented attachment. Science," Science, 336(2012), 1014-1018.

[10] M. King, W. F. McClure, L. C. Andrews: Powder diffraction file alphabetic index, Inorganic Phases/Organic Ohases, International Center for Diffraction Data: Newtown Square, PA, 1992.

[11] Y. M. Fazil Marickar , P. R. Lekshmi, L. Varma, P. Koshy. "Elemental distribution analysis of urinary crystals," Urol. Res., 37 (2009), 277-282.

[12] L. A. Thurgood, R. L. Ryall. "Proteomic analysis of proteins selectively associated with hdroxyapatite, brushite, and uric acid crystals precipitated from human urine," J. Proteome Res., 9(2010), 5402-5412. 